John Richardson

\title{
Metamorphoses by Philip Glass: some psychological and ethnomusicological observations
}

In this paper I will examine the recent composition Metamorphoses (composed 1988) by Philip Glass making use of techniques from both ethnomusicology and the psychology of music. ${ }^{1}$ I will attempt to show in practice that techniques developed in ethnomusicology for the holistic analyses of music as a cultural phenomenon and the findings of cognitive musicology pertaining to elementary perceptive and generative thought processes are not diametrically opposed, but when combined can provide insights concerning the work of art that would otherwise be difficult to obtain. My premise is that our understanding of music is complex and multifaceted, involving dynamic interaction and uninhibited synthesis between diverse modes of thought. Thus, the subsymbolic thought processes with which the literature in cognitive psychology has largely been concerned and the higher symbolic thought processes which have been the main focus of attention in cognitive ethnomusicology are viewed as interdependent and frequently inseparable in terms of actual experience. The symbiotic nature of this relationship is, of course, acknowledged by a growing body of literature in both ethnomusicology and Western musicology, although analyses of contemporary music explicitly combining both are still few and far between. Philip Glass's music provides an ideal model for this type of research, combining as it does an interesting palette of subtle psychological effects as well as implicitly and explicitly defined cultural extensions.

1) I would like to express my gratitude to Philip Glass for taking the time to discuss his music with me. I would also like to thank the following people; Pirkko Moisala, Erkki Salmenhaara and Yrjö Heinonen, all of whom have had some influence on the material printed here at various stages during the research process. 


\section{Background}

Metamorphoses was composed in 1988, released on the Solo Piano recording in 1989 , and is commonly acknowledged by critics as one of the more successful of Philip Glass's postminimal compositions. In 1988, the composer was commissioned by the New York State Council to perform a series of five solo concerts, after which he embarked on a tour of twenty cities in the United States with only his hand luggage as company. Solo performance offered Glass a sense of freedom not possible when performing with his ensemble or when working with a large theatrical company:

Touring by myself is very easy. With the ensemble there is about two tons of equipment to transport as well as eleven people. But when I travel by myself, there is no tour manager and I carry no music - it's all in my head. (Glass, interview in Dalton 1989, 2.)

Performance is, however, a familiar experience for Glass - as is the case with most of the other composers that have come to be associated with the minimalist movement. He began his compositional career in the 1960s performing music in attics and art galleries in the fashionable SoHo district of New York and has continued to perform with The Philip Glass Ensemble since that time. The Solo Piano live performances were an opportunity to experience the more intimate audience contact of a chamber music, rather than an orchestral or theatrical performance situation in which composer/audience interaction is mediated, at least partially, by the interpretations of the other performers.

In the Solo Piano project, as in the performance art of fellow New Yorkers Laurie Anderson and Meredith Monk, the roles of composer and performer were combined resulting in a direct experience for the listener of the composer as a physically tangible - fellow human being. As with the performance artists who employed minimal techniques in their music, the presence of the composer in the performance situation represents a clear deconstruction of the Mind/Body distinction, which feminists have been working hard to dismantle because of the mapping of the sexes (Male/Female; Mind/Body) onto it (see MacCormack \& Strathern 1980; and McClary 1991). The act of performance by the composer thus confirms, in no uncertain terms, his physical as well as his intellectual and expressive faculties, and complements the music which resounds in kinetic energy as well as rationalistic order.

The performance situation is conveyed to the listener unable to attend a performance in person by the cover of the compact disc. In addition to explicit "program notes" including information on the origin of the musical material used in the recording and its original performance contexts, the visual imagery used reveals a great deal concerning the music it is packaging. A black and white photograph portrays Glass sitting at the keyboard of a grand piano. The overriding effect of the photograph (which is in fact predominantly black, except for the right 


\section{Richardson}

half of Glass's face, a reflection on the piano, and the text-graphics) is one of subdued nostalgia. Glass's expression is reflective and somewhat solemn. The book shelf in the background reveals that it was probably taken in Glass's home study and creates an informal and intimate atmosphere (the effect would have been very different if he had been seated in a concert hall in full evening dress). The style of the text is elegant but uncomplicated; the letters are a simple white on a black background. The atmosphere invoked by the packaging of the CD is, we shall see, in many respects allied in characteristics with the music it is packaging.

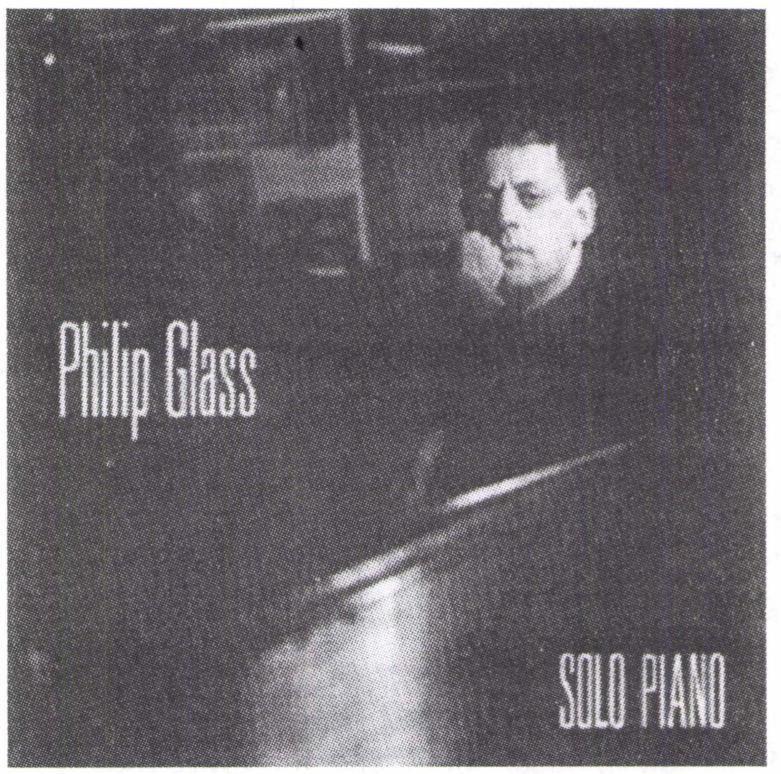

Front cover of Solo Piano compact disc (1989)

Although Glass has characterised much of the music on this recording as romantic (Dalton 1989, 2), it differs in some important respects from piano music typical of the romantic period, the primary distinction being that Solo Piano is, on the whole, a non-virtuosic collection of works. The written music to this collection is commercially available (unlike many of Glass's other works) and presents no great difficulty to even a pianist of relatively limited technical ability. As Jody Dalton has observed in the sleeve notes of the recording, it harks back to "the fading American tradition of the piano as a familiar, shared experience of simple, tuneful music." (Ibid.)

Despite its essentially non-virtuosic character, the influence of romantic music is clearly perceivable in Glass's writing for the piano. His exploitation of the colouristic textural possibilities of the instrument is, for example, evocative of Frédéric Chopin's piano music. Like Chopin, Glass explores a particular colouristic effect in each section or song, and his melodies are graceful and unpretentious. The pianistic nature of the work reveals a keen awareness of the various stages of the history of keyboard music in the Western music tradition while retaining a distinct contemporary feel. Glass himself cites Schubert as an influence in this particular work (Glass, interview with the author 9.9.1993). Echoes of J.S. Bach are also perceivable if one listens, for example, to the first Prelude from the first book for the Well-Tempered Clavier - its gradual organic change and rhythmic repetition certainly mark this as a minimalist piece some 250 years be- 
fore the term was applied to music. More recently La Monte Young has shown in his Well Tuned Piano (recorded in 1987) that the piano is an excellent medium for repetitive music, allowing as does a rich series of harmonic overtones to resonate in sympathy with any given chord when the sustain pedal is applied. Glass exploits fully the possibilities of the instrument in Metamorphoses creating a subtle and pervasive aura of rich harmonic sonority. In this case, the written music provides only a limited reduction of what the listener actually hears, the sonic reality being an elaborate fabric of intertwining voices far more complex and profound than knowledge of the played notes alone would suggest (see the spectrogram analyses at end).

Metamorphoses is a synthesis of two of Glass's earlier works rearranged for solo piano. The title is taken from Kafka's short story of the same name ${ }^{2}$ and numbers three and four are derived from the incidental music he wrote for a play based on that story. Although some parallels can be found between the plot of the short story and Glass's composition, I am nevertheless of the opinion - and there is little evidence to suggest otherwise - that the work is not intended to be a direct programmatic reference to Kafka's work. The title was retained because of its general abstract nature, because it is descriptive of the compositional processes employed in the work, and perhaps because it is easily compatible with both Eastern and Western thought. ${ }^{3}$ The piece that follows Metamorphosis on the Solo Piano recording, Mad Rush, was composed for the occasion of the Dalai Lama's (one of the highest Tibetan Buddhist gurus) first public address in New York City, in autumn 1981, and was originally written in open-ended form (i.e., without a beginning or an end) to reflect the centrality in Buddhist thought of perpetual change; a fact that may shed some light also on the composition that precedes it.

The second work from which the musical material of Metamorphosis is drawn is Glass's soundtrack to the Errol Morris film The Thin Blue Line. In the sleeve notes, the original context of the film music is explained for the benefit of the listener:

The Thin Blue Line ... depicts the true story of a man's wrongful

2) In the short story, also known as The Transformation, the protagonist, Gregor Samsa wakes to find himself transformed into an insect. The rest of the story concerns itself with how Gregor and the Samsa family cope with their predicament. The situation proves to be intolerable for both parties and relief is found only when Gregor (the insect) dies following a gradual deterioration in his health. It is then that a second transformation occurs. The Samsa family who had grown to be overly dependent on Gregor, find that they can manage for themselves without him. His child sister is transformed into a women, his obsequious father begins to stands up for himself, and his mother's health improves. The story, therefore, begins and ends with a transformation, although the greatest transformation of the two is arguably the spiritual transformation of the Samsa family at the end of the story.

3) Metamorphosis is a pervasive theme in Greek mythology, and this is undoubtedly how Kafka originally conceived of the idea for his short story. Transformation is in many cultures symptomatic of animistic religious thought and can be extended to a wide variety of animate and inanimate objects, serving as a philosophical allegory to underline the ultimate unity of all matter as well as the inherent divisions and conflicts of human life. It is noteworthy that sexual transformations and ambiguity are also at the centre of both Greek mythology and Indian folklore (Geertz 1983, 81-85; Irving 1990, 149179.): a fact that may add some weight to a gendered reading of the present work. 
conviction for the murder of a Dallas police officer. The touching melody of number two and the diatonic harmonies throughout provide an ironic counterpoint to the film's numerous reenactments of the shooting. The thrice repeated two-note theme (a descending minor third) in numbers one and five recalls the movie's pathetic litany of interviews and testimonies. (Dalton 1989, 3.)

Once again, I am of the opinion that the above exposition is not in its present context intended as a program, although the fact that it was included in the sleeve notes of the recording suggests that it may reveal something of the ideational origins of the musical material. It should be remembered, for example, that, even though the core of the musical material in these sections was inspired by the pathos of the dramatic action in the film, the overall effect of the piano arrangement is somewhat different to that of the original orchestral version.

Although Metamorphosis has its origins in diverse compositional material, Glass has, as the title suggests, succeeded in "transforming" it into one of his the most structurally coherent and deeply contemplative works. In Metamorphoses one, two, three, four and five Glass carries on where he left of in the piano Opening of Glassworks, with its delicately interwoven polyrhythmic voices and gently shifting modal harmony. Here Glass maintains the balance and poise of the earlier work but adds to it the knowledge of larger structures and range of expression he has gained through his work in music-theatre.

\section{Micro-structures: simple triads or subtle harmonic paradox? (bars 1-5)}

Although Metamorphoses is considerably more sophisticated in terms of structure than Glass's earlier more rhythmically orientated and predominantly modal minimal works, it is nevertheless useful to divide the composition into a fundamental two-tiered structure for the purpose of analysis. Traces of the chaconne, applied more rigidly in earlier compositions (Satyagraha, Akhnaten, Songs from liquid days) as well as more recent works (Concerto for Violin and Orchestra, 2nd movement), surface in this compositions in a more subtle manner. In terms of bass ostinati or harmonic formulae it would certainly be difficult to accept such a definition, notwithstanding the scarcity of traditional four-bar chaconne cadences. Nevertheless, the way the music works is somewhat similar. As in the chaconne the focus is largely on micro-level changes within the cadence rather than motivic development or dramatic juxtapositions related to material outside it. In psychological terms, therefore, it useful to think of the chaconne as a perceptual norm from which the present composition deviates in certain significant respects. The usefulness of such a standpoint will become more apparent as the analysis progresses.

We shall see as the analysis proceeds that the transformations of musical material implied in the title take place within a loose cyclical framework. It follows from this that the central axle and the composition and its beginning and end are 
liable to be of overt structural import to the internal coherence of the composition. Numbers one and five are virtually identical and, as such, provide the frame for the cyclical development that occurs between these two poles.

The opening five bars of number one form an eloquent initial proposition, namely a transition from the initial $E$ minor triad to a highly ambiguous sixth degree chord (see computer analyses at the end). This is executed primarily be means of a descending bass ostinato $(1-7-6)$ which anticipates the later melodic theme of descending thirds. This opening five-bar phrase is highly enigmatic. The listener expects the chaconne-like sequence to resolve to a dominant chord but this expectations is denied. The listener is denied even the relative harmonic stability of the subdominant tonal function. Instead the transition is accomplished (although it could be regarded primarily as a linear transition) by means of the modal mediant and submediant chords. Some harmonic impetus is provided, however, by this sequence, as the mediant chord, G major, is also the secondary dominant of the submediant chord, C major-seventh $(-3)$, which it precedes.

The final chord of the sequence is virtually impossible to categorise in unambiguous terms. It can be interpreted as a dominant (flattened) seventh (-5) constructed on the sixth-degree or as an Italian augmented sixth chord - which is itself ambiguous as it can be interpreted both as an altered fourth- and as an altered sixth-degree chord. If it is interpreted as the latter, then, according to historical convention, it should resolve to the dominant. No such resolution occurs; the first conventional (leading-note) cadence is withheld until part three of the composition(!). The chord can also be interpreted as a tacit reference to the AfroAmerican tradition; in blues and rock it is common practice to increase harmonic interest by adding a flattened seventh to the major triad (even to the tonic). I do not consider it necessary or desirable to provide an unequivocal explanation of this chord as it is held that ambiguity is a central tenet of Glass's musical language. Moreover, it seems appropriate that one is divided between an interpretation founded in the Western classical tradition and one founded in the AfroAmerican vernacular tradition; even if this ambiguity between high and popular culture was not intended by the composer, I suspect that he would not be entirely displeased by it. The enigmatic sixth-degree chord is, therefore, simply left hanging (it is followed by a quarter-note rest), and returns unresolved to the tonic.

Probably the most cogent explanation, however - and one that I have become convinced is the most accurate from the perceptual standpoint - can be attained by examining Glass's own analysis of an earlier composition, the opera Einstein on the Beach. The phrase below appears also in altered form in the other two portrait operas, Satyagraha and Akhnaten, and is the unifying musical element of the entire trilogy. It would seem on first hearing to be a traditional cadential formula until one takes into account the altered or pivotal chord that occurs in middle which results in a closing chord that is a semitone lower than one would normally expect. Glass's own analysis of the cadence is as follows: 


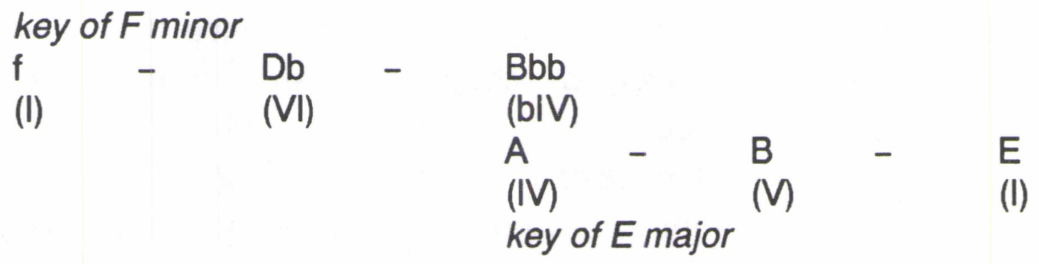

Glass's own analysis of Einstein on the Beach cadence (from Glass 1987, 60)

When approached in this way the opening bars of metamorphosis one, which were virtually impossible to categorise in conventional tonal terms, can be more readily understood. The initial $E$ minor is transformed into the mediant $G$ by simple bass movement à la chaconne. $G$, which is the mediant in the key of $E$ minor is also the dominant chord in the key of $C$. This does not, however, explain why instead of arriving at a conventional $C$ major triad we arrive at $C$ with a flattened seventh and no fifth. I think the above interpretations of conventional Western and Afro-American harmonic practices are useful here as they no doubt shape the listener's expectations to a certain extent. The final answer to the paradox (or koan, to use the Buddhist term), though, is to be found in bars 47-52, where the initial phrase is repeated but with what looks like a French augmented sixth (or perhaps a Jazz eleventh?) added on the end. Although one would certainly expect such a chord to be followed by the dominant in its normal tonal context, in metamorphosis the two upper voices ( $\mathrm{F \#}$ and $\mathrm{Bb}$, or $\mathrm{A \# )}$ ) slip in effortless parallel movement up one semitone to $G$ and $B$ respectively. Thus the answer to the riddle is really quite simple: there is no need to look for a "dominant" because there is none. Furthermore, there is no need for one. Instead there are two interdependent tonal centres each of which is relatively stable and becomes more so through repetition.

Of course when we talk in the Western tradition of setting up two parallel key centres the examples that come most readily to mind, from this century at least, are those of Stravinsky and Milhaud. However, the paradigm example of bitonality, Stravinsky's famous "Petrushka chord" in which C and F\# chords are heard simultaneously and which can be regarded largely as independent of one another, is one of jarring dissonance. Although Glass acknowledges the influence of both of these composers - and it is noteworthy that Milhaud was one of his teachers - he clearly thinks of bitonality in different terms in his own compositions:

Well of course I was (influenced by Milhaud) but I don't think it sounds very much like him. It's a very different approach to it. It's not so much about setting up two keys at the same time, which is what those composers did, but we're treating a situation where a piece can be viewed as being in more than one key at the same time. In other words, it's kind of (like), an example is those kind of optical illusions where you can look at a stair going one way or you can look at it going the other way but you really can't look both ways. So basically, you can hear it in 
one tonality with added tones or you can hear it in another tonality with added tones but the idea is not to hear them going on at the same time. But I did study with Milhaud, I like him very much, I like his music, but that's a much "notier" way of writing music. (Glass, interview with the author, 8.9.1993)

The principle Glass is identifying is one very similar, in terms of perception, to that which John Sloboda has noted regarding contrapuntal lines in music. Sloboda's hypothesis is that harmonic music is perceived as an ambiguous pattern capable of ground-figure reversal. In contrapuntal music "focal attention" can only be directed at one line at a time while the others act as background. The listener may notice that something is wrong if the line he or she is not listening to is incorrect (i.e., does not follow the rules governing that particular music style) but will not be able to ascertain the exact nature of the anomaly unless focal attention is redirected towards the offending line. (Sloboda 1985, 168-170) I would suggest that with bitonality in Glass's music a similar perceptual process is involved. The tonal ground for one's perceptions is governed by the initial key centre until an increasing amount of evidence supporting a second key centre forces the listener to shift her perspective. Glass's example of reversing stairs provides a valid analogy for the cognitive processes involved in listening to music constructed from such principles. Regardless of whether one begins looking at the diagram below from above or below, at some point one is forced to shift one's perspective to make sense of the picture.

It is perhaps no co-incidence that Glass first conceived of this technique while writing the opera Einstein on the Beach. A parallel between the "tonal relativity" implicitly suggested in this technique and Einstein's Theory of Relativity is not difficult to perceive. Just as Einstein's theory of relativity forced scientists to relinquish from Newtonian concepts of space and time as objectively fixed and absolute, so the simultaneous presence of two tonalities - neither of which imposes upon the clarity and internal order of the other but both of which rely structurally upon their twin - highlights the relativity and subjectivity of tonality. It should be noted also that the optical illusion described by Glass was exploited by the Dutch artist M.C. Esher's in his lithograph "relativity" and in other more recent works of art employing optical illusions (such as in the above drawing by Sandro del Prete). That this idea could be extended to include cultural relativity offers also a very real foundation for a socially founded reading. Glass comments:

It's in the operas that these ideas really get developed...the requirements of dramatic presentation often require you to invent language which then becomes more generally used. I mean, that's true of the history of opera generally, if you look at Monteverdi or if you look at Wagner. The transformation of the language came about because of dramatic reasons but then once that language was achieved then it was used in a more general way (Glass, interview with the author, 8.9.1993) 


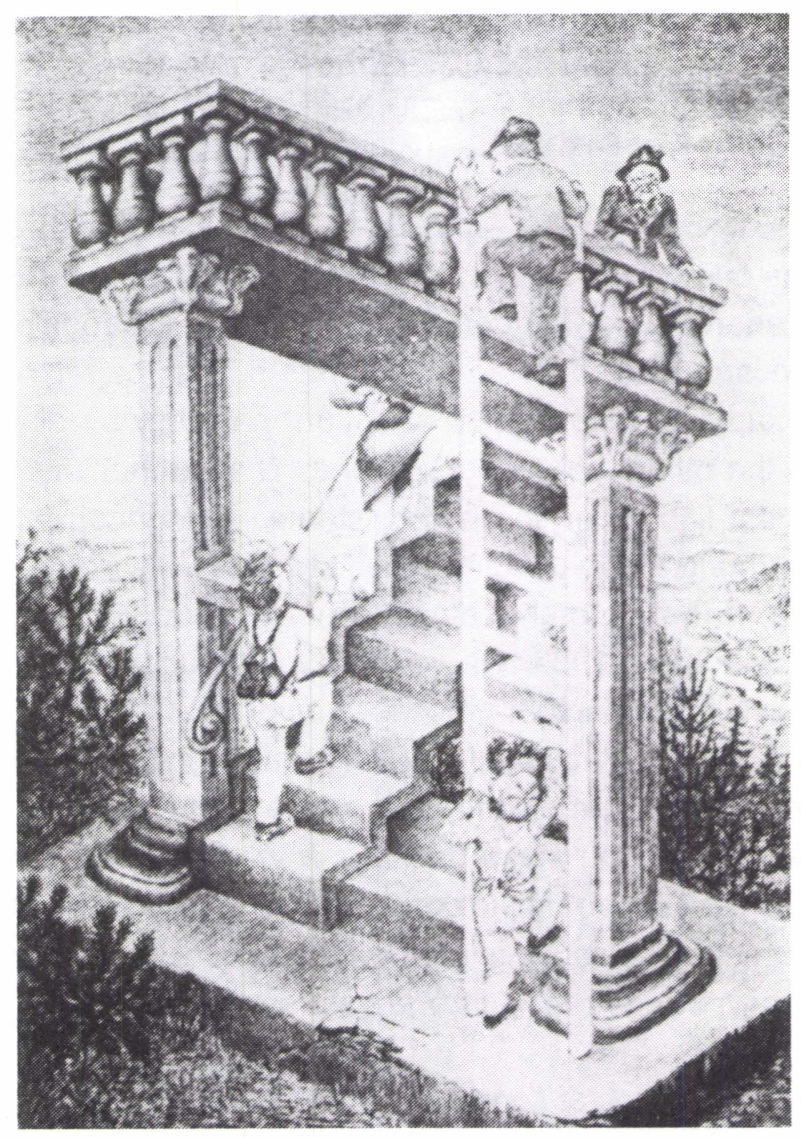

Sandro del Prete: "Winding staircase up to Belvédère II", pencil drawing (in Ernst 1986, 90).

The same psychological principle of "focused" perception can perhaps also be extended to include polyrhythmic music such as found in Metamorphosis three and to a lesser extent in the highly syncopated pattern employed in four, where right and left hand rhythms are disjuncted by one eighth note. When playing, and perhaps listening to polyrhythmic music with two parts it is certainly difficult to focus on both parts at the same time. One of them ultimately becomes the "focal" and the other the "background" part. In performance one is required to switch and co-ordinate between the two by means of "coincidences" in the divergent rhythmic patterns. For the performer, it is only when the fingers have acquired sufficient motoric control and the brain inter-nalised the polyrhythmic pattern and switched to a more intuitive mode of consciousness that the music can be enjoyed without selfconscious concentration.

The harmonic ambiguity of the opening five bars is also characterised by a certain degree of rhythmic ambiguity; as neither of the two "tutti" chords in each bar is consistently stressed (see computer analysis of the sound waveform envelope), the listener is left in some doubt as to whether the downbeat falls on the initial quarter-note or on the dotted half-note which follows. The overall effect of this opening passage is, because of its lack of resolution and ambiguity analogous to an unanswered question, or in terms of Eastern thought is comparable to the linguistic paradoxes, or koans, used in Zen Buddhism. Through these the paradoxical nature of reality is accentuated, the purpose being to encourage a level of awareness that transcends the limitations of language. The spiritual, or meditative nature of the music is strengthened further by the rich array of harmonic overtones that resonates in sympathy with each played note (see spectrogram analysis). 


\section{Macro-structures: transformation and cyclical form}

The harmonic development which began in number one continues throughout the composition. The initial five-bar harmonic sequence (viewed in conventional tonal terms, i $\mathrm{Wl} \mathrm{VI7} \mathrm{It} \mathrm{VI),} \mathrm{extended} \mathrm{by} \mathrm{one} \mathrm{bar} \mathrm{(Fr} \mathrm{VI)} \mathrm{towards} \mathrm{the} \mathrm{end} \mathrm{of} \mathrm{number} \mathrm{one}$ as well as in the final recapitulation, transforms itself into ever more complex manifestations. In number two, it is divided into two phrases (i MI VI VH : VI VH i), which should more properly, perhaps, be understood in bitonal terms because of the flattened seventh degree G7 and its secondary dominant C, although the sense of goal direction is increased - and the sense of bitonality thus weakened - by the diatonic harmonic movement towards the tonic in the second phrase. Number three reverts to conventional leading-note harmony (i III iv NII VII V i) - augmented now to span sixteen bars - and fluctuates between major and minor modes (I H). Number four contains the strongest cadential resolution (i + iv NII :

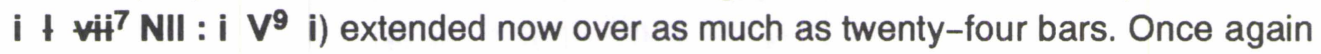
this can be viewed in traditional tonal terms apart from the interchangeability between minor and major chords, which itself arises here primarily because of linear rather then harmonic movement. From here, one is returned to the second manifestation of the opening sequence in number five.

The arc-like form is paralleled also in the melodic development. Number one is characterised by a haunting two-note motive of descending thirds. In number two, this is elaborated and extended in accordance with the concomitant harmonic development. In numbers three and four the need for melodic interest is superseded by the tension created by the polyrhythm of the former and the strict syncopated disjunction of the later as well as by the tensions of conventional leading note harmony. Number five returns the listener to the elegiac simplicity of the opening theme and to contemplative "other-worldliness" of Glass's bitonality. Because of the lack of resolution of the opening sequence (reiterated in its later manifestation in number five) as well as its frail minor-major bimodality, it is the antithesis of number three in which resolution to the major mode, to conventional tonality, and to the altered dominant of the macro-level chaconne sequence, finally occurs. It is highly significant, however, that at the end of the composition we are returned to the enigmatic opening passages. There is no final resolution; only uncertainty, sensitivity, but at the same time a profound and somewhat paradoxical sense of resignation and introspective peace.

A characteristic feature of Philip Glass's approach to composition is his ability to deal with larger macro-level harmonic interrelationships in a coherent manner without losing track of micro-level gradual change. Just as on the micro-level, the tonal centres of the five metamorphoses together form a chaconne-based cadential sequence (i - iv - $\left.{ }_{b} \mathbf{V i i}{ }_{b} \mathbf{V I I}{ }_{b} \mathbf{V i i}-\mathbf{v i}-\mathbf{i}\right)$ similar to the less sophisticated macro-level plagal cadence of Changing Opinion (opening song of Songs from liquid days) and a similar plagal cadence - described by Gregory Sandow as a gigantic "Amen" - from which the Gandhi opera Satyagraha is constructed 
(Sandow 1982, 95). The opera Akhnaten is similarly enshrouded within the key of A minor and The Making of the Representative from Planet Eight, based on Doris Lessing's novel of the same name, like Metamorphoses is made up of an extended cadence around the key of E minor (Swed 1988, 578).

It is interesting to consider how Glass conceives of these extended cadences in terms of tonality: can it be thought of as a kind of polytonality when the listener is aware of both the macro-level tonality of the entire composition and the microlevel tonal centre at the same time? In Changing Opinion from the Songs from Liquid Days song cycle, for example, Glass exploits this dualism ingeniously, constructing entire sequences that can be interpreted from two divergent key centres.

\begin{tabular}{|c|c|c|c|c|}
\hline $\begin{array}{l}\text { Thus, the sequence, } \\
\text { can also be heard as: }\end{array}$ & $\begin{array}{l}f: \\
\text { c/C: }\end{array}$ & iv & bVIII & $\begin{array}{l}v \\
\text { I }\end{array}$ \\
\hline $\begin{array}{l}\text { And the sequence, } \\
\text { as: }\end{array}$ & $\begin{array}{l}\text { f: } \\
\text { c/C: }\end{array}$ & iv & $\begin{array}{l}\text { VI } \\
\text { NII }\end{array}$ & $\begin{array}{l}\text { i7 } \\
\text { iv7 }\end{array}$ \\
\hline
\end{tabular}

Glass comments:

I don't know (if he would categorise that as polytonality). I think there must be some notion of that though it's not one I've articulated very much. A lot of these ideas are more experiential than analytical. That's why it's a little difficult for me (to say), but I would agree that I think that's the case, especially in that piece (Akhnaten). The feeling of $A$ minor is almost always there somewhere. (Glass, interview with the author, 8.9.1993)

In Metamorphoses, the technique of sequence is employed on the macro-level to provide a sense of momentum and structural unity between its various parts; thus, the initial tonal centre of $E$ minor found in number one drops by two steps of a perfect fifth, to $A$ minor in number two and then $D$ minor alternating to $D$ major in number three, before returning via the altered (or major) sixth degree of $C$ minor, in number four, to the original key of $E$ minor in number five. Once again, this harmonic interrelatedness is, as we have seen, complemented by tempo, rhythm and other micro-level changes. Whereas the central axle of Songs was founded on the sub-dominant, in Metamorphosis it is the altered dominant, as manifested in both major and minor flattened seventh-degree chords, that forms the apex of the arc.

As was the case in Songs, the central axle itself is symmetrical in form (in this case $\mathbf{a} \mathbf{b}$ a b a: $\left.{ }_{\mathbf{b}} \mathbf{V i i}{ }_{\mathbf{b}} \mathrm{VII}_{\mathbf{b}} \mathbf{V i i}_{\mathbf{b}} \mathrm{VII}_{\mathbf{b}} \mathbf{v i i}\right)$, thus reinforcing the impression of concentric circles as the listener draws closer and pulls away from this section. Its structural import is strengthened further by the first appearance of conventional leading-note harmony in the composition (prior to this point, the unaltered or flattened seventh degree of the natural minor scale is the preferred form) - in addition to alternation between major and minor modes - in contrast with the rest of the 
composition which is in the minor mode throughout. The effect of the shift from the minor to the major mode at this point is, as a result of its singularity, stunning (or climatic?). Glass himself notes that the shift from major to minor in the same key was inspired mainly by Schubert and indeed this influence is not difficult to discern (Glass, interview with the author, 8.9.1993).

\begin{tabular}{|c|c|c|c|c|c|c|}
\hline Part One & Part Th & Part Three & & rt Four & \multicolumn{2}{|c|}{ Part Five } \\
\hline i & iv & bvii bVII bvii bVII bvii & vi & i & tonality & \\
\hline$d=108-112$ & $d=96-104$ & polyrhythm: $d=132 / d=88$ & \multicolumn{4}{|c|}{$d=120-130 d=108-112$} \\
\hline$d=120$ & & & & & $d=120$ & tempo \\
\hline 5:39 & 7:19 & $5: 30$ & & $7: 00$ & 5:03 & duration \\
\hline $\mathbf{A}$ & B & C & & D & $\mathbf{A}^{\prime}$ & form \\
\hline
\end{tabular}

Macro-level asymmetrical symmetry in Metamorphosis

Number three is distinguished further from the other metamorphoses because of its polyrhythmic texture; although it is written in 6/8 time, it is actually the left hand only that is playing in this rhythmic configuration. The right hand plays two dotted quarter-notes a bar throughout, creating a strong tension between perceived binary and ternary parts. It is worth noting that while the left hand is playing the fastest tempo of the entire composition $(d=132)$, the right hand is playing the slowest $\left(d_{.}=88\right)$. This is a second ambiguity to add to the minor/major ambiguity described above; once again it occurs at the central fulcrum of the composition.

Although number four contains some polyrhythmic passages, it is not polyrhythmic throughout; interest is preserved in this section, however, by its strict rhythmic syncopation $(4 / 4 . d \downarrow d \downarrow d$ ) which is related rhythmically to the syncopated opening phrases of the composition $(4 / 4 \quad \cdot d .)^{4}$. Number three is, thus, by far the most complex and dynamic as well as the most rhythmically ambiguous of all five metamorphoses as experienced by the listener.

Elsewhere, I have hypothesised that a parallel with mandala symbolism can be recognised here (Richardson, 1993a; 1993b). I would certainly be reluctant to give up this hypothesis entirely, given that Glass is a practising Tibetan Buddhist who more likely than not uses mandala diagrams as a tool in meditation. That visual forms used to induce a meditational condition could have a subliminal influence on the forms used to construct music which is meditative by nature does not seem unlikely to me. Helen Semyonova has revealed similar mandala-like

4) The effect of this strong right hand syncopation $(4 / 4, d \ldots d \delta)$ when superimposed on the more stable left hand pattern $(4 / 4 \mathrm{~d} d d d)$ is very similar to the polyrhythm of the previous section, and brings to mind also of the canonic phase shift pactices of Steve Reich. 
principles of construction in the compositions of Schnittke, Denisov and Shchedrin (Semyonova 1993), minimalists Terry Riley and Peter Michael Hamel have both written circular musical scores analogous to Buddhist and Hindu mandala diagrams, and the British composer John Tavener has written a number of cyclical compositions analogous to icons used in orthodox christianity. Mandalas employed in Tantric Buddhism are constructed from a circle at the centre of which is a highly ambiguous central axle from which all transformations emanate. There are some obvious parallels which I will not pursue further here, but I am certainly of the opinion that such an interpretation as well as a gendered or socially based "hearing" would certainly be grounded in these circumstances, particularly when the more general context of Glass's theatrical works is taken into account.

It should be noted, though, that Glass himself has conceived of this particular piece more in linear terms: "they're five pieces that seem to be music in the process of transformation in a very gentle way" (Glass, interview with the author, 8.9.1993). In this respect, a more obvious visual parallel would by the series of works by the Dutch artist M.C. Escher (see illustrations on this and the opening page) which carry the same name as the Glass composition and in which certain formal similarities can be easily discerned: these include gradual transformation and cyclic form. 

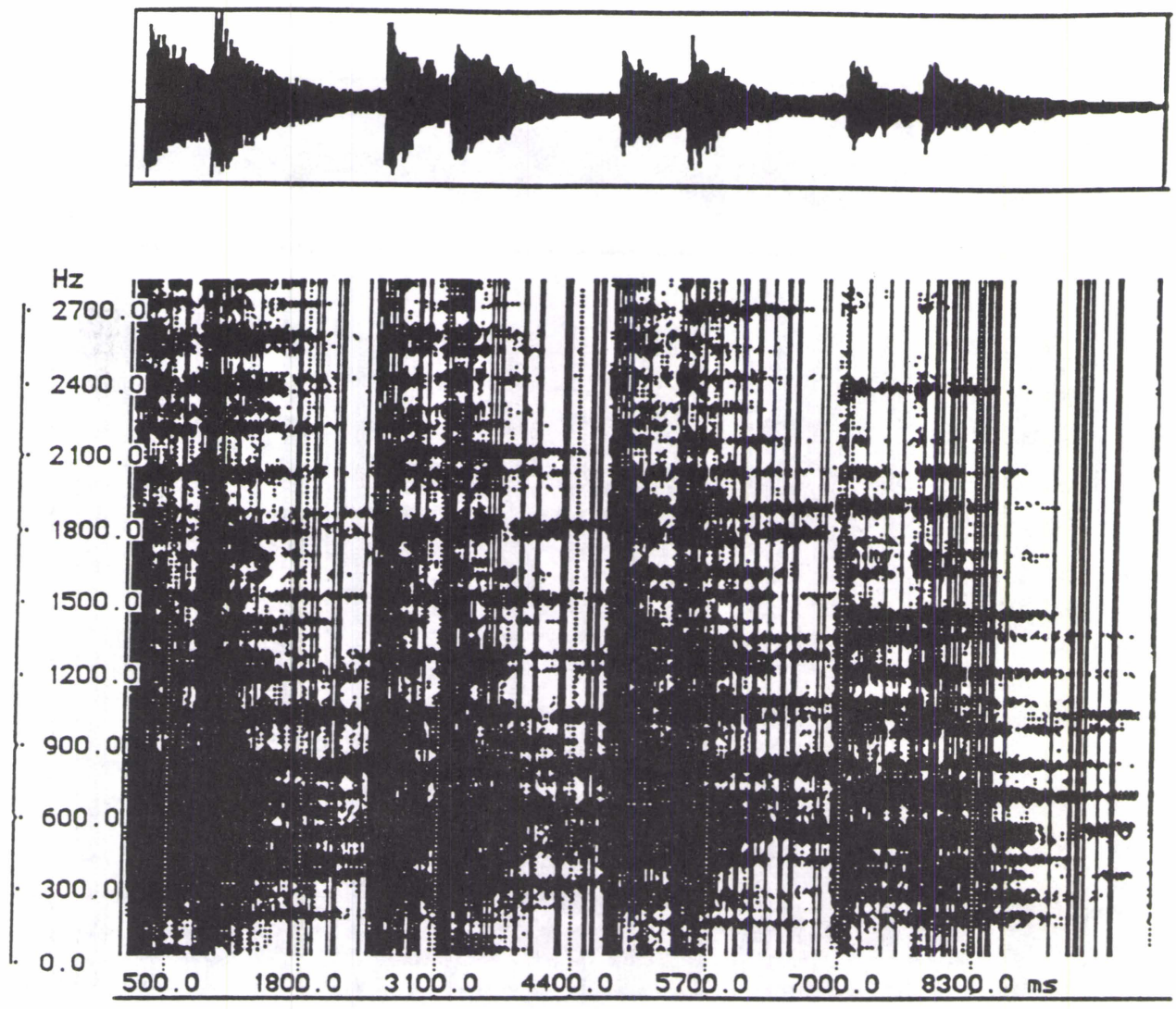

Moderate $1_{\bullet}=108-112$ )

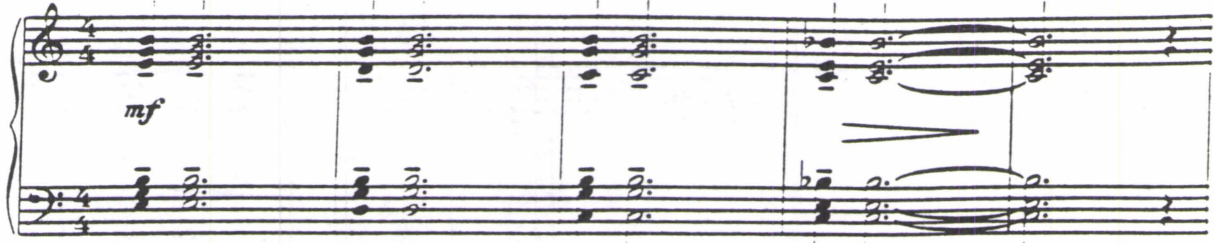

Computer analysis of Metamorphosis I, (bars 1-5)using SignalyzeTM 2.41. program with Macintosh hardware

Above: sound waveform envelope illustrates an approximation of the amplitude of the wave form. The computer analysis shows that there is no consistent stress on either beat, which, when perceived, calls into question which of the two is in fact the downbeat.

Middle: Spectrogram analysis (very slimband) demonstrates the rich overtone structure of the opening proposition.

Below: musical notation of the same four bar phrase: 

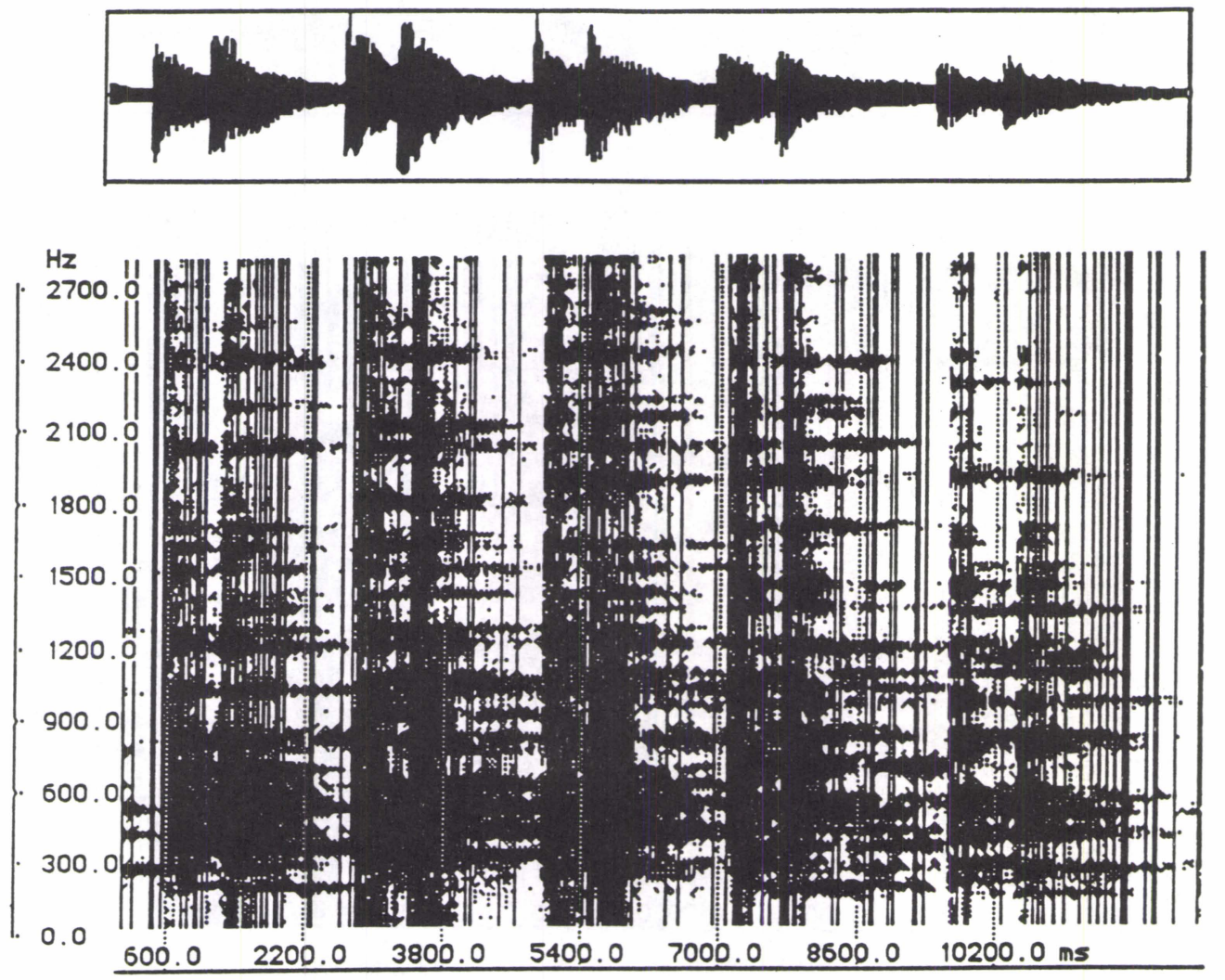

$\therefore \quad(.=108.112)$

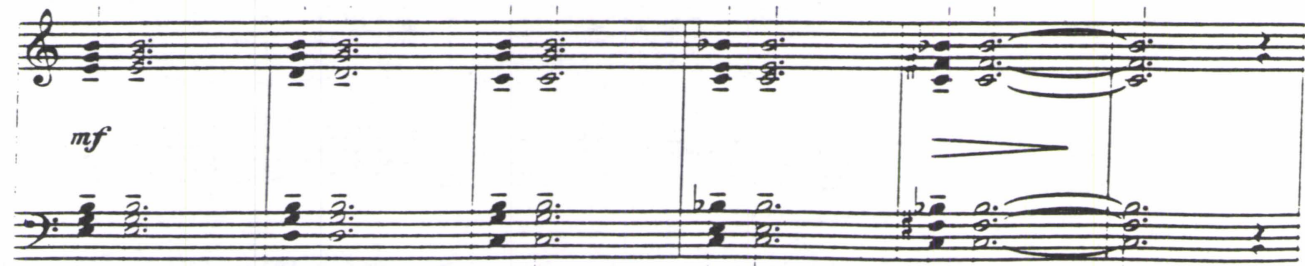

Computer analysis of Metamorphosis I, (bars 47-52) 


\section{References cited}

Dalton, Jody 1989. Sleeve notes to Solo Piano by Philip Glass. CBS Records

Ernst, Bruno 1978. M.C. Escherin taikapeli Berlin: Taco

- 1986. The eye beguiled: optical illusions Köln: Benedikt Taschen

Geertz, Clifford 1983. Local knowledge: further essays in interpretative anthropology New York: Basic Books Inc., Publishers

Glass, Philip 1987. Opera on the beach: Philip Glass on his new world of music theatre London: Dunvagen

- 1988. Metamorphoses sheet music, New York: Dunvagen Music Publishers (used with permission)

Irving, Forbes 1990. Metamorphosis in Greek myths Oxford: Clarendon Press

McClary, Susan 1991. Feminine Endings: music, gender, and sexuality Minnesota: University of Minnesota Press

MacCormack, Carol \& Marilyn Strathern 1980. Nature, gender and culture Cambridge: Cambridge University Press

Richardson, John 1993a. Music as mandala: ambiguity and androgyny in "Changing Opinion" and Metamorphosis" by Philip Glass Licentiate dissertation for the University of Jyväskylä, Finland

- 1993b. Music, gender and cultural cognition in Proceedings of the First International Conference on Cognitive Musicology (editors, Jouko Laaksamo \& Jukka Louhivuori) Jyväskylän yliopiston monistuskeskus: Jyväskylä, Finland

- (primary source) interviews with Philip Glass, 8.9.1993 and 9.9.1993

Sandow, Gregory 1982. The uses of structure Village Voice 13 January 1982 (p. 95)

Semyonova, Helen 1993. On some non-traditional forms of variability in contemporary music in Proceedings of the First International Conference on Cognitive Musicology (editors, Jouko Laaksamo \& Jukka Louhivuori) Jyväskylän yliopiston monistuskeskus: Jyväskylä, Finland

Sloboda, John A. 1985. The musical mind: the cognitive psychology of music Oxford: Clarendon Press

Swed, Mark 1988. Philip Glass's operas The Musical Times November 1988 (pp. 577579)

Glass, Philip: Selected discography

- Akhnaten 1983. CBS Masterworks series, M2K 42457

- Concerto for violin and orchestra Deutsche Grammophon $437091-2$

- Einstein on the Beach 1975. CBS Masterworks series, M4 38875

- Satyagraha 1979. CBS Masterworks series, 13M 39672

- Solo Piano 1988. Includes Metamorphosis, Mad Rush, and Wichita Sutra Vortex. CBS MK 45576

- Songs from liquid days 1985. Includes Changing Opinion, lyrics by Paul Simon, Lightning and Freezing, lyrics by Suzanne Vega, Liquid days (part one), Open the Kingdom (liquid days part two), lyrics by David Byrne, Forgetting, lyrics by Laurie Anderson CBS FM 39564 